\title{
Social Loafing in Brainstorming CMC Teams: The Role of Moral Disengagement
}

\author{
Omar Alnuaimi \\ University of Arkansas \\ oalnuaimi@,walton.uark.edu
}

\author{
Lionel Robert \\ University of Arkansas \\ lrobert@walton.uark.edu
}

\author{
Likoebe Maruping \\ University of Arkansas \\ $\underline{\text { lmaruping@walton.uark.edu }}$
}

\begin{abstract}
Social loafing, i.e. the tendency of some individuals to not exert as much effort in team settings as when they are working alone, has been identified as a major source of productivity loss in brainstorming teams. Studies of social loafing in brainstorming Computer Mediated Communication teams are scant. This paper examines the mechanisms through which previously identified antecedents (Group size and perceived loafing of other members) of social loafing work. This paper utilizes the Theory of Moral Disengagement which helps explain how people engage in antisocial, i.e. social loafing in this case, behavior by disengaging their self-sanctions that otherwise will restrain such conduct. To test the hypotheses, this study employs a controlled experiment with 47 undergraduate students from a Middle Eastern university. Findings indicate that diffusion of responsibility and dehumanization mediates the positive effect of group size on social loafing in brainstorming teams. Also, attribution of blame was found to have a direct negative effect on social loafing. Implications of these findings are discussed and managerial guidelines presented.
\end{abstract}

\section{Introduction}

Organizations are increasingly relying on teams that utilize information and communication technologies (ICTs) to increase creativity within organizations $[8,11,14]$. Sixty percent of professional employees work in teams that communicate primarily through ICTs [20]. These teams allow organizations to combine the ideas of individuals located across temporal and geographic boundaries [17]. Despite the advantages associated with these teams they also present new challenges to corporations. One of the challenges relates to the use of ICTs as a primary medium of communication (see [33] for media differences).

Traditional control and coordination-such as direct supervision, geographic collocation, and shared experiences-are often missing in ICT-mediated communication environments $[17,31]$. Social loafing, defined as the tendency of individuals to put less effort in team settings than when they work individually [8, 23], has been identified as a major problem associated with a lack of control and coordination in traditional collocated teams. Social loafing has been linked to several negative team outcomes such as lower performance, lower group cohesiveness, lower satisfaction, and increased absenteeism [13, 24]. Social loafing is expected to be a larger problem in teams that communicate through ICTs $[8,27]$.

Despite the long history of research on brainstorming, idea generation, in computer-mediated communication (CMC) teams [11], the problem of social loafing has not received enough attention from IS researchers [8]. Past research on social loafing in CMC teams has identified team size and perceived social loafing of others as key antecedents [18]. While the identification of these antecedents has been valuable, there is a need for a deeper understanding of the underlying mechanisms that help predict social loafing behavior. For example, team size is often driven by organizational needs and necessities and, therefore, cannot be easily modified. Hence the importance of understanding why and how those antecedents influence social loafing in CMC teams.

The main objective of the current research is to identify the cognitive mechanisms that mediate the effect of team size and perceived loafing of other team members on social loafing. To this end, we employ the theoretical lens provided by the Theory of Moral Disengagement [2]. Drawing on this theory, we identify three mediating variables: diffusion of responsibility, dehumanization, and attribution of blame. We begin by discussing the theoretical lens and its relevance to the research problem. This is followed by the development of theoretical arguments supporting the proposed mediational role of moral disengagement constructs. Then we present the methodology used to test the proposed model. Results 
of the study are presented and theoretical and practical implications are offered.

\section{Theoretical Background}

The theory of moral disengagement [2] is useful for understanding why individuals in brainstorming $\mathrm{CMC}$ teams engage in social loafing. Viewed through this theoretical lens, social loafing is understood as an inappropriate or antisocial behavior in which a group member withholds contributions to the team despite the fact that he/she required to work up to their optimal performance regardless of whether: they are working alone or in a group, their task is visible or not, and they are working in a small or a large group. Another assumption here is that group members are aware of their social loafing behavior.

Bandura [2] proposed the idea of moral disengagement as an individual difference that influences people's moral decision making. According to Bandura [2], people are guided by personal standards of ethical behavior and most people tend to refrain from acts that violate their own moral standards. An individual's moral evaluation is translated into actions through two self-regulatory mechanisms of moral agency: social sanctions and self sanctions. Social sanctions are theorized to restrain antisocial or immoral behaviors through the expectation that such behaviors will result in "social censure" and other adverse social consequences. However, social sanctions are limited because of the fact that most immoral behaviors go undetected. Yet, people will still refrain from engaging in transgressions because of "self-sanctions" or self condemnation.

"As long as self sanctions override the force of external inducements behavior is kept in line with personal standards. However, in the face of strong external inducements, such conflicts are often resolved by selective disengagement of self-sanctions. This enables otherwise considerate people to perform selfserving activities that have detrimental social effects." [3 p.280]

We believe that the theory of moral disengagement constitutes an adequate theoretical lens for examining social loafing in brainstorming CMC teams. This is because increased anonymity and decreased social presence in those teams is expected to weaken the role of social sanctions that would otherwise restrict antisocial acts. When the role of social sanctions is weakened, self-sanctions or self condemnation become more salient. The theory of moral disengagement focuses on those self sanctions and how individuals override them in order to perform antisocial or inappropriate behaviors, such as social loafing.

\section{Theory and Research Model}

\subsection{Group Size}

Ringelmann was the first to note that group size has a positive effect on social loafing [22]. In the $19^{\text {th }}$ century, he found that collective performance of a group pulling a rope is inferior to what should have been expected based on prior individual performance. $\mathrm{He}$ also noted that individuals' effort in group tasks decrease as group size increases and individuals' contributions become less visible. As groups become larger, visibility of individuals' effort decreases and monitoring of individuals becomes more difficult $[1$, 19].

From a rational choice perspective, increased group size will increase social loafing because individuals would feel that their individual contribution would be less crucial to group success than in a small group [21]. Therefore, they would expect less sanction by coworkers and supervisors if they are not putting forth effort, as opposed to individuals in small groups, who would expect their contribution to be more critical to group success, and who would expect their coworkers and supervisors to sanction them if they are not providing the expected contribution. Another factor that was proposed as an explanation for the positive effect of group size on social loafing is the difficulty in coordinating effort as group size increases [25].

\section{H1a: Group size will be positively related to individual's social loafing.}

\subsection{Diffusion of Responsibility}

As mentioned earlier, group members might have a perception that large group size would make individuals' effort more dispensable [21]. In their experiment using a cognitive task, Weldon and Mustarri [36] found that group members who believed they shared responsibility with another member "used less information when evaluating a decision than members who believed they were the solely responsible for the task".

Diffusion of responsibility for conduct weakens the exercise of moral control by obscuring personal agency [6]. "When every one is responsible, no one really feels responsible" [4 p. 365]. As group size increases, more people are involved in accomplishing 
the group's goal, and, hence, responsibility is diffused among a larger number of stake holders. This makes it easier to engage in social loafing without the fear of activating self sanctions, since personal agency for group goal achievement is obscured.

H1b: Individuals' perception of diffusion of responsibility will mediate the relationship between group size and individual social loafing.

\subsection{Dehumanization}

Arguments underlying theories of group dispersion and social loafing are consistent with the notion that people are restrained from engaging in antisocial behavior due to their fear of social sanctions. However, these theories ignore the idea that people are also restrained from antisocial behavior because of self sanctions or self condemnation. Unless individuals disengage self-sanctions from their inappropriate conduct, they do not engage in such conduct [5]. Since we established earlier that social loafing is an antisocial act, people need to disengage self-sanctions before loafing.

One set of disengagement mechanisms operates on the recipient of the antisocial behavior, i.e. other group members in our case. Because the strength of selfsanctions depends in part on how the recipients are viewed, this moral disengagement mechanism, referred to as dehumanization, operates through divesting people of human qualities. Perceiving another as human increases perceived similarity and, therefore, makes it difficult to target them with antisocial behavior [4]. As group size increases, the human part of members becomes less salient and individuals in brainstorming $\mathrm{CMC}$ teams might feel that they are interacting with a nameless crowd rather than individuals within a team. Such perceptions and feelings would deactivate the self-regulatory function because it fosters a perception of dissimilarity between the performer (i.e. individual) and the recipients (i.e. other group members). Milgram [29] noted that most people persistently refuse to behave punitively when the situation is personalized [4]. Therefore, when a team member does not see other members (as in the case of many CMC teams) and team size increases, then it would be easier for him/her to target them with antisocial behaviors, such as social loafing. Hence, we hypothesize:

H2a: Individuals' perception of humanity of other group members will mediate the relationship between group size and social loafing.

\subsection{Perceived Loafing by Others}

A perception that other group members are loafing would lead to an increase in social loafing [7, 10, 16, and 32]. Jackson and Harkins [16] found that individuals exerted less effort when they believed that other group members are not trying hard in a shouting task. Likewise, Schnake [34] found that individuals decreased their contribution when they believed that other group members were withholding effort. Robinson and O'Leary-Kelly [32] found a positive relationship between the level of antisocial behavior exhibited by coworkers and an individual's own antisocial behavior. It is the perception of coworker loafing that matters, regardless of actual behavior [30]. It is expected that such perceptions would be higher in the context of CMC team, where task visibility decreases as members usually do not see each other while performing the task.

\section{H3a: Perceived loafing of other team members will be positively related to individuals' social loafing.}

The impact of perceived loafing by others will, in part, be mediated by the attribution of blame. In order for the individual to engage in social loafing, he/she needs to selectively disengage their internal controls that otherwise would stop them from performing the antisocial behavior. The relevant moral disengagement mechanism in the case where coworkers are loafing is attribution of blame. An individual who feels that other coworkers are loafing will blame them for driving him/her to loaf. By blaming others, team members are more inclined to believe that their "injurious actions" are not only excusable but "selfrighteous" [4 p.366].

H3b: Attribution of blame will mediate the relationship between perceived loafing by coworkers and social loafing.

\section{Methodology}

Forty seven undergraduate business students from a Middle Eastern university participated in this study. Eleven brainstorming teams - six comprised of three members and the other five of six members - were formed using random assignment. The average age was 24.26 (std. Deviation is 3.72) and $23 \%$ of the subjects were females. Course credit was offered for participating and everyone who participated in the study received the same amount of course credit. 


\subsection{Task}

We used a brainstorming task that was validated in prior research [18]. It required the groups to generate ideas to solve the university's parking problem. According to McGrath's [28] taxonomy of tasks, this is a Type 2 task that calls for idea generation and creativity. Parking is a persistent and pervasive issue on university campuses and is quite relevant and frustrating to students since most of them have their own cars and drive to campus. As a worm-up exercise, students worked individually on a brainstorming task that required them to generate uses for a knife and a toothbrush [35]. Then they were asked to work in teams and respond to the following request: "You have probably tried to find a place to park around campus and know that it is not always easy. Even if you don't have a car on campus, you probably have witnessed such problems. This is especially true when you are late for class, an appointment or a ball game. The question put forth to you today is: What can be done to help reduce the parking problem? Be specific, complete and concise - yet you need to provide enough information so that someone else can fully understand your idea without requiring further explanation".

\subsection{Experimental Setting}

Students were seated in two computer labs. Computers were distributed in a $U$ shape in which each user faced the wall. Students were asked to leave an empty PC between each two users and not to talk to, or look at, other students during the task.

\subsection{Technology}

All groups used a chat system that was based on PHP technology. We developed a PHP based chat system in which each team had their own assigned virtual room and were not allowed access to any other room. The chat system provided an archive of all the chat session transcripts. One of the researchers provided technical support by showing the students how to use the system.

\subsection{Procedure}

Students were welcomed to the lab and were asked to sit at assigned computer stations. A researcher welcomed them and asked them to read the consent form and sign it if they agreed to participate. Then each student was given a slip of paper that has a name (an alias) that they would use during the experiment. The aliases given included names like $\mathrm{A} 3, \mathrm{C} 4$, and
M2; where the letter represents the team name while the number represents the individual. Each student was then asked to work individually for 15 minutes and generate as many uses as $\mathrm{s} / \mathrm{he}$ can for a knife and a toothbrush. Students wrote their ideas on sheets of paper that were collected from them at the end of the 15 minutes. They were then told to launch an Internet browser, go to a specific website, and click on their team's name. Doing that would launch a chat room for the specific team. Students used their assigned names in the chat room and not their real names. The group task lasted 20 minutes. Following the group task, all students were asked to fill out a questionnaire that captured their perceptions about the model variables, i.e. diffusion of responsibility, dehumanization, attribution of blame, and other member's perceived loafing. , Finally, students were thanked for their participation, debriefed and dismissed.

\subsection{Measures}

Social loafing was measured by the number of ideas generated by each individual during group discussion. A lower number of ideas indicated a higher level of social loafing. This way of measuring social loafing is consistent with prior research, e.g. [8] and [35]. Diffusion of responsibility (DFR), dehumanization (DHM), and attribution of blame (ATB) were all measured using some newly developed items with most items adapted from [4]. For example, Diffusion of responsibility items included (I had limited responsibility toward achieving the team's objective). Dehumanization items include (I felt that I was interacting with a computer rather than a human being). Attribution of blame items included (Targets of antisocial behavior usually do things that make them deserve such behavior). Perceived loafing of others (OSL) was measured with items adapted from [13]. for example, one item used to measure perceived loafing of others says (Some members of my team were less likely to generate ideas when another member was available to do this task).

\subsection{Analysis Technique}

We used partial least squares (PLS), a structural equation modeling (SEM) technique, to analyze relationships between variables in the research model (for more information on PLS, see [15]). The software used was SmartPLS V 2.0.M3. Because PLS uses a component-based approach, it places minimal requirements on sample size and residual distributions [26]. All constructs are modeled with reflective indicators. 


\section{Results}

PLS, analysis involves two steps: 1) analysis of the measurement model; and 2) analysis of the explanatory and predictive power of the model. Details of each step are outlined below.

\subsection{Measurement Model Results:}

Descriptive statistics for the research constructs are shown in Table 1. We assessed the psychometric properties of the scales by examining item loadings, discriminant validity, and reliabilities. Item loadings and reliabilities above .70 are considered acceptable [12]. Table 1 reports the composite reliability scores and inter-construct correlations. All constructs have acceptable internal consistency since all reliability scores are above .70 . The composite reliability scores are more than adequate, ranging from .83 for dehumanization to .94 for perceived loafing of others.

\subsection{Structural Model Results}

We ran the structural model in SmartPLS2 to obtain the path coefficients and corresponding tvalues. PLS path coefficients are interpreted as standardized beta weights in a regression analysis. We followed Baron and Kenny (1986) approach for testing the mediation effects. First, we tested the direct effect of team size and perceived other's loafing on number of ideas. We included the number of ideas generated by individuals in the pre-task session as a control. The path coefficients, $t$-values and explained variances for the direct effect model are shown in Figure 1. H1a

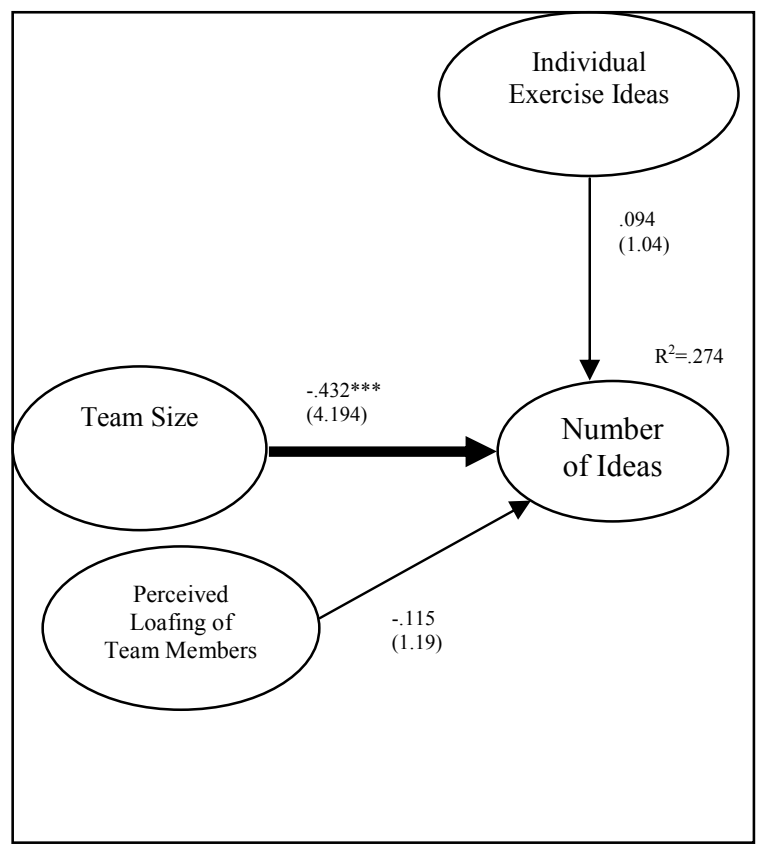

Figure 1. Direct effects model

Note:* $\mathrm{p}<.05 ; * * \mathrm{p}<.01 ; * * * \mathrm{p}<.001$. Bold lines indicate significant relationships.

posits that group size will be positively related to individuals' social loafing. This hypothesis is supported. The coefficient of the direct path is -.432 (tvalue $=4.194, \mathrm{p}$-value $<.001)$. The negative coefficient indicates that as team size increases, number of generated ideas per individual decreases. H3a posits that perceived loafing of other members will be positively related to individuals' social loafing. We did not find support for this hypothesis since the path coefficient between the two constructs is only -.115 (t-

Table 1. Reliability and inter-construct correlations

\begin{tabular}{|l|c|c|c|c|c|c|c|c|c|c|}
\hline & Mean & $\begin{array}{c}\text { Std. } \\
\text { Dev. }\end{array}$ & Rel. & ATB & DHM & DFR & NOI & OSL & SSL & SIZ \\
\hline $\begin{array}{l}\text { Attribution of Blame } \\
\text { (ATB) }\end{array}$ & 4.88 & 1.47 & 0.90 & 0.845 & & & & & & \\
\hline Dehumanization (DHM) & 2.07 & 1.01 & 0.83 & -0.098 & 0.755 & & & & & \\
\hline $\begin{array}{l}\text { Diffusion of } \\
\text { Responsibility (DFR) }\end{array}$ & 4.72 & 1.51 & 0.90 & $0.428^{* *}$ & $0.300^{*}$ & 0.812 & & & & \\
\hline Number of Ideas (NOI) & 2.45 & 1.28 & 1.00 & 0.284 & $-0.486^{* * *}$ & $-0.393^{* *}$ & 1.000 & & & \\
\hline $\begin{array}{l}\text { Perceived Loafing of } \\
\text { Other Memebers (OSL) }\end{array}$ & 4.39 & 1.71 & 0.94 & 0.021 & 0.259 & $0.375^{* *}$ & $-0.284^{*}$ & 0.868 & & \\
\hline $\begin{array}{l}\text { Self-reported Loafing } \\
\text { (SSL) }\end{array}$ & 3.50 & 1.73 & 0.84 & 0.141 & 0.185 & $0.406^{* *}$ & -0.228 & $0.551^{* *}$ & 0.855 & \\
\hline Team Size (SIZ) & 4.36 & 1.56 & 1.00 & 0.097 & $0.430^{* *}$ & $0.671^{* * *}$ & $-0.502^{* * *}$ & $0.408^{* *}$ & $0.294^{*}$ & 1 \\
\hline
\end{tabular}

Shaded values are the square root of the average variance extracted. It shows the variance shared between a construct and its measures. Shaded diagonal elements should be larger than off-diagonal elements in order to satisfy discriminant validity requirement. 


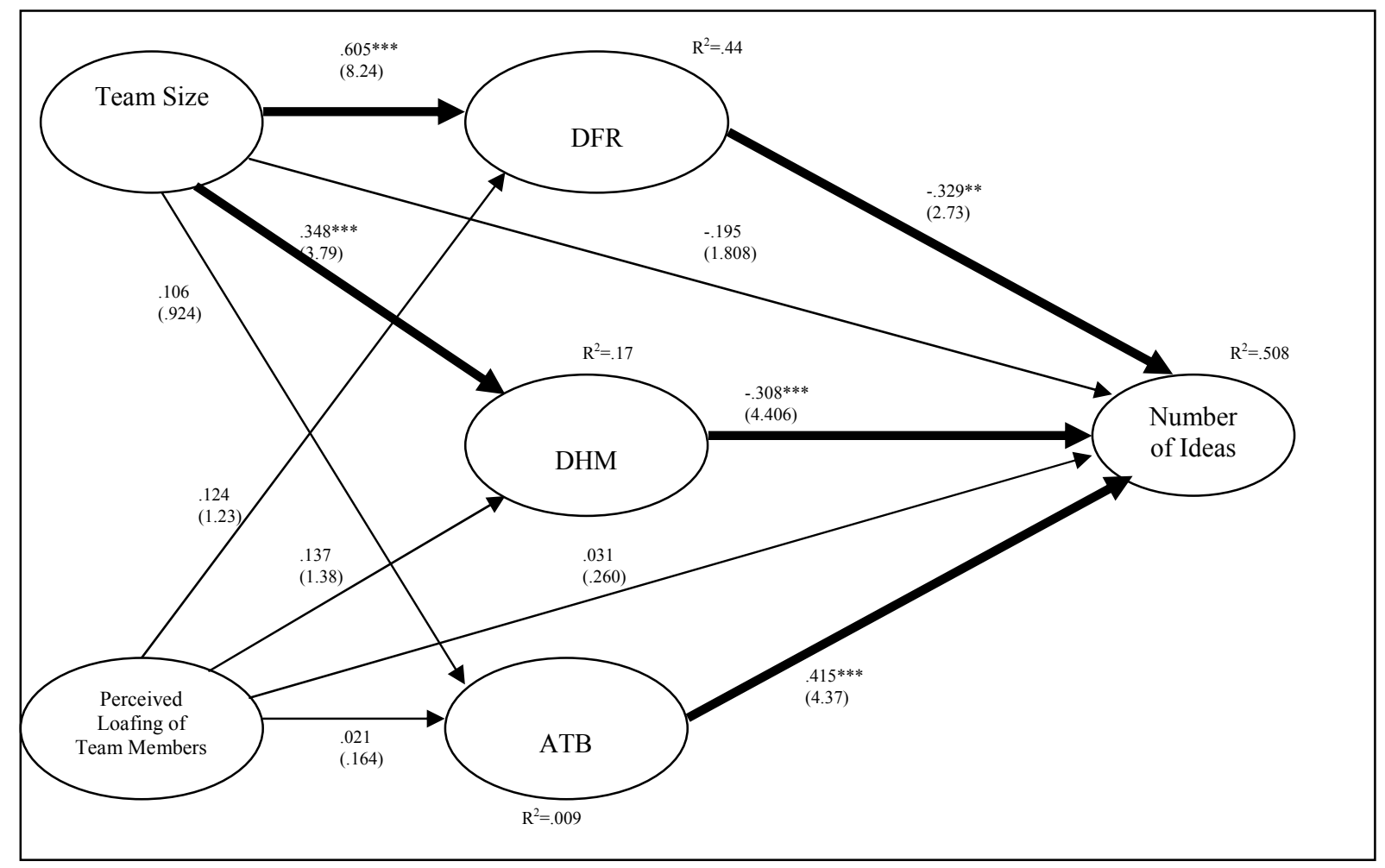

Figure 2: Mediational effects model

Note: $* * \mathrm{p}<.01 ; * * * \mathrm{p}<.001$. Bold lines indicate significant relationships.

value $=1.19, \mathrm{p}$-value $>.1$ ). Also, we found no significant relationship between number of ideas generated in the pre-task session and the number of ideas generated in the team session. Therefore, we decided to exclude this control variable from further analysis.

Figure 2 shows the path coefficients, t-values and explained variances for the mediational effect model are shown in Figure 2. Since the current study is intended to be a pilot study, we ran the model with all possible relationships. H2a, which posits that individual's perception of diffusion of responsibility (DFR) will mediate the relationship between group size and individual's social loafing, is strongly supported. Coefficients of the paths from group size to DFR and then to social loafing are .605 ( $\mathrm{t}$-value $=$ 8.24 , p-value $<.001$ ) and -.329 ( $\mathrm{t}$-value $=2.73$, $\mathrm{p}$-value $<.01$ ), respectively. The negative coefficient between diffusion of responsibility (DFR) and number of ideas indicates that individuals with high perception of DFR will generate significantly fewer ideas than those with low perception of DFR. Group size explains about $43 \%$ of variance in individuals' DFR.

Dehumanization was hypothesized to mediate the relationship between team size and social loafing (H2a). The path coefficient between dehumanization and number of ideas is -.308 (t-value $=4.406$, $\mathrm{p}$-value $<.001)$. The negative coefficient indicates that individuals with high perception of dehumanization will generate significantly fewer ideas than those with low perception of this construct. We found a significant relationship between team size and dehumanization. The path coefficient is .348 (t-value $=3.79$, $\mathrm{p}$-value $<.001$ ). This finding indicates that the larger the group size is, the higher the perception of dehumanization, therefore $\mathrm{H} 2 \mathrm{a}$ was supported.

In addition, we did not find support for H3b which posits that attribution of blame will mediate the relationship between perceived loafing by co-workers and social loafing. The path coefficient between perceived loafing of others and attribution of blame is not significant $(\mathrm{t}$-value $=.164)$. However, we found a significant relationship between attribution of blame and number of ideas (t-value $=4.37$, p-value $<.001$ ), although the relationship was in the opposite direction. The hypothesis suggested a negative relationship between the two constructs but we found a positive coefficient of .415 . We will elaborate more on this contrary-to-hypothesis finding in the discussion section. Our model explained about $50 \%$ in the variance of number of ideas, which we used as a proxy for social loafing. 


\section{Discussion}

The objective of the current research was to identify the cognitive mechanisms that mediate the effect of team size and perceived loafing of other team members on social loafing within brainstorming CMC teams. We employed the theoretical lens provided by the theory of moral disengagement [2] to identify three mediating variables. First, the current research found that diffusion of responsibility fully mediated the effect of group size on social loafing. Individuals in larger groups had higher perceptions of diffusion of responsibility and therefore, produced fewer ideas than those in smaller groups. As Bandura noted, "when everyone is responsible, no one really feels responsible" [4 p. 365]. We found that dehumanization was a significant antecedent of social loafing. Individuals who did not perceive the interactions to be personalized generated fewer ideas than those with low level of dehumanization perceptions. Third, we found support for attribution of blame as an antecedent of social loafing, but the mediation role of this construct was not supported in this sample. We found that individuals who were blaming other members for the low performance of their group were, in fact, contributing more ideas. This finding is contrary to our earlier hypothesis. It can be explained using the theory of social compensation, which states that people will work harder collectively than individually when they expect their co-workers to perform poorly on a meaningful task [37]. Therefore, it is possible that members who were blaming others for their poor performance were also trying to socially compensate for the low performance.

As with all research, the current study has some limitations. The study's sample is limited to undergraduate students enrolled in a business school at a Middle Eastern university who were using a chat system to perform the task. The generalizability of the research findings to subjects in other settings maybe somewhat limited. For example, social norms of different cultures might have an effect on social loafing and thus our sample is limited to the Middle Eastern culture in which we ran the experiment. Also, our sample is composed of only business students. Usually, brainstorming teams are interdisciplinary in order to have different perspectives from different backgrounds. Therefore, the current research needs to be replicated to examine the robustness of the findings across different samples, cultures, types of teams, and technologies. Also, we had a small sample size. To identify the minimum sample size required, we followed [9] recommendation by multiplying the number of paths leading to the endogenous construct with the most paths leading into it by 10 . The (Number of Ideas) construct has five paths leading into it. Therefore, a minimum sample of 50 would be enough. Since we have 47 participants; we conclude that we almost have an adequate sample size for running the model. Future work will address this limitation by having a much larger sample size. Another limitation is related to the length of the experiment. The whole experiment lasted for about one hour. As such, we might not have given enough time for the team members to develop some of the perceptions such as their perceptions of loafing of other members. Future studies should examine those perceptions over a longer period of time. Finally, we did not measure students' motivation to participate. Students might have not generated ideas because they are not motivated to do so. However, since we randomly assigned students to teams, randomization should take care of the role of student's motivations. Future studies should pay attention to students' motivation to participate. Also, future studies investigating social loafing in brainstorming teams may want to investigate the role of media choice in influencing teams' productivity. For example, the use of richer media such as video conferencing might lower the perceptions of dehumanization and thus decrease social loafing.

Despite such limitations, the current study has important implications for teams' research in general and CMC team research in particular. It is one of the first studies to identify the cognitive mechanisms through which team structure variables, e.g. size and perceived loafing of co-workers, affect social loafing. Although some researchers have speculated about the role of diffusion of responsibility as a possible explanation for the effect of group size on social loafing, we are not familiar of any study that operationalized and tested it in the context of social loafing. Additionally, social loafing researchers would benefit from our attempt to identify and operationalize the three mediators, i.e. diffusion of responsibility, dehumanization, and attribution of blame. By revealing the mechanisms through which previously identified antecedents affect social loafing, researchers can move forward to design interventions that work on those mechanisms. We also contribute to the social psychology literature by extending the theory of moral disengagement and integrating it with social loafing theories. By viewing loafing as an antisocial and immoral behavior we are opening the doors for researchers to extend theories developed originally for analyzing immoral conducts and use them as theoretical lenses for examining loafing behavior.

Since most organizations utilize teams that communicate primarily through ICTs, lessons learned 
from this study will be very useful for practitioners. Our findings confirmed that the size of a team matters, and that adding more members to teams increases the likelihood that members will be contributing less toward team goals. However, this effect takes place only through its influence on members' perceptions of diffusion of responsibility and dehumanization. Managers of brainstorming CMC teams need to make changes to decrease these perceptions. For example, they might want to emphasize the responsibility of each member toward accomplishing the task. Also, they might ensure that the communication medium is more human, probably by using richer media that increases the social presence of team members such as video conferencing. Organizations need to weigh the costs of collocation of teams against the costs of losses in productivity due to increased dehumanization and hence social loafing.

\section{Acknowledgements}

The authors wish to thank Dr. Abdelaziz Khamis (Ajman University) for his help in running the experiment. Also, Thanks are extended to the faculty members and $\mathrm{PhD}$ students at the University of Arkansas - Fayetteville for their valuable insights on this research.

\section{References}

[1] Albanese, R., and van Fleet, D.D. "Rational Behavior in Groups: The Free-Riding Tendency," Academy of Management Review (10:2), 1985, pp 244-255.

[2] Bandura, A. "Mechanisms of Moral Disengagement," in: Origins of Terrorism: Psychologies, Ideologies, Theologies, and States of Mind, W. Reich (ed.), Erlbaum, Hillsdale, NJ, 1990, pp. 161-191.

[3] Bandura, A. "Social Cognitive Theory of SelfRegulation," Organizational behavior and human decision processes (50:2), 1991, pp 248-287.

[4] Bandura, A., Barbaranelli, C., Caprara, G.V., and Pastorelli, C. "Mechanisms of Moral Disengagement in the Exercise of Moral Agency," Journal of Personality and Social Psychology (71:2), 1996, pp 364-374.

[5] Bandura, A., Caprara, G.V., Barbaranelli, C., Pastorelli, C., and Regalia, C. "Sociocognitive SelfRegulatory Mechanisms Governing Transgressive Behavior," Journal of Personality and Social Psychology (80:1), 2001, pp 125-135.

[6] Bandura, A., Underwood, B., and Fromson, M. "Disinhibition of Aggression through Diffusion Of Responsibility And Dehumanization Of Victims," Journal of Research in Personality (9:4), 1975, pp 253-269.

[7] Campion, M.A., Papper, E.M., and Medsker, G.J. "Relations between Work Team Characteristics and Effectiveness: A Replication and Extension," Personnel Psychology (49:2), 1996, pp 429-452.

[8] Chidambaram, L., and Tung, L.L. "Is Out of Sight, Out of Mind? An Empirical Study of Social Loafing in Technology-Supported Groups," Information Systems Research (16:2), 2005, pp 149-168.

[9] Chin, W. "The Partial Least Squares Approach to Structural Equation Modeling," in: Modern Methods for Business Research, G.A. Marcoulides (ed.), Lawrence Erlbaum Associates, Mahwah, NJ, 1998.

[10] Comer, D.R. "A Model of Social Loafing in Real Work Groups," Human Relations (48:6), 1995, p 647.

[11] Dennis, A.R., Wixom, B.H., and Vandenberg, R.J. "Understanding Fit and Appropriation Effects in Group Support Systems via Meta-Analysis," MIS Quarterly (25:2), 2001, pp 167-193.

[12] Fornell, C., and Larcker, D. F. "Evaluating Structural Equations Models with Unobservable Variables and Measurement Error," Journal of Marketing Research (18:1), 1981, pp 39-50.

[13] George, J.M. "Extrinsic and Intrinsic Origins of Perceived Social Loafing in Organizations," Academy of Management Journal (35), 1992, pp 191-202.

[14] Griffith, T.L., Sawyer, J.E., and Neale, M.A. "Virtualness and Knowledge in Teams: Managing the Love Triangle of Organizations, Individuals, and Information Technology," MIS Quarterly (27:2), 2003, pp 265-287.

[15] Hulland, J.S. "Use of Partial Least Squares (PLS) In Strategic Management Research: A Review of Four Recent Studies," Strategic Management

[16] Jackson, J.M., and Harkins, S.G. "Equity in Effort: An Explanation of the Social Loafing Effect," Journal of Personality and Social Psychology (49:5), 1985, pp 1199-1206.

[17] Jarvenpaa, S.L., Knoll, K., and Leidner, D.E. "Is Anybody Out There?: Antecedents of Trust in Global Virtual Teams," Journal of Management Information Systems (14:4), 1998, pp 29-64.

[18] Jessup, L.M, T Connolly, T. and J Galegher "The Effects of Anonymity on GDSS Group Process with an Idea-Generating Task," MIS Quarterly, 14:3, 1990, pp. 313-321.

[19] Jones, G.R. "Task Visibility, Free Riding, and Shirking: Explaining the Effect of Structure and Technology on Employee Behavior," Academy of Management Review (9:4), 1984, pp 684-695.

[20] Kanawattanachai, P., and Yoo, Y. "Dynamic Nature of Trust in Virtual Teams," Journal of Strategic Information Systems (11:3-4), 2002, pp 187-213.

[21] Kidwell Jr, R.E.a., and Bennett, N. "Employee Propensity to Withhold Effort: A Conceptual Model to Intersect Three Avenues of Research," Academy of Management Review (18:3), 1993, pp 429-456.

[22] Kravitz, D.A., and Martin, B. "Ringelmann Rediscovered: The Original Article," Journal of Personality and Social Psychology (50:5), 1986, pp 936-941.

[23] Latané, B., Williams, K., and Harkins, S. "Many Hands Make Light Work: The Causes and Consequences of Social Loafing," J. Personality Soc. Psych. (37), 
1979, pp 822-832.

[24] Liden, R.C., Wayne, S.J., Jaworski, R.A., and Bennett, N. "Social Loafing: A Field Investigation," Journal of Management (30:2), 2004, p 285.

[25] Littlepage, G.E. "Effects of Group Size and Task Characteristics on Group Performance: A Test of Steiner's Model," Personality and Social Psychology Bulletin (17:4), 1991, p 449.

[26] Lohmoller, J. Latent Variable Path Modeling With Partial Least Squares Physica-Verlag, Heidelberg, 1989.

[27] Majchrzak, A., Malhotra, A., and John, R. "Perceived Individual Collaboration Know-How Development through Information Technology-Enabled Contextualization: Evidence from Distributed Teams," Information Systems Research (16:1), 2005, pp 9-27.

[28] McGrath, J.E. (1984). Groups: Interaction and performance. Englewood Cliffs, NJ: Prentice-Hall.

[29] Milgram, Stanley. "The Perils of Obedience." (abridged from "Obedience to Authority: An Experimental View," 1974)

[30] Mulvey, P.W., and Klein, H.J. "The Impact of Perceived Loafing and Collective Efficacy on Group Goal Processes and Group Performance," Organizational Behavior and Human Decision Processes (74:1), 1998, pp 62-87.

[31] Piccoli, G., and Ives, B. "Trust and the Unintended Effects of Behavior Control in Virtual Teams," MIS Quarterly (27:3), 2003, pp 365-395.

[32] Robinson, S.L., and O'Leary-Kelly, A.M. "Monkey See, Monkey Do: The Influence of Work Groups on the Antisocial Behavior of Employees," Academy of Management Journal (41:6), 1998, pp 658-672.

[33] Robert, L.P. and Dennis, A.R. Paradox of Richness: A Cognitive Model of Media Choice, IEEE Transactions on Professional Communication, 48:1, 2005.

[34] Schnake, M.E. "Equity in Effort: The "Sucker Effect" In Co-Acting Groups," Journal of Management (17:1), 1991, pp 41-55.

[35] Shepherd, M.M., Briggs, R.O., Reinig, B.A., Yen, J., and Nunamaker Jr, J.F. "Invoking Social Comparison To Improve Electronic Brainstorming: Beyond Anonymity," Journal of Management Information Systems (12:3), 1995, pp 155-170.

[36] Weldon, E., and Mustari, E.L. "Felt Dispensability in Groups of Coactors: The Effects of Shared Responsibility and Explicit Anonymity on Cognitive Effort," Organizational Behavior and Human Decision Processes (41), 1988, pp 330351.

[37] Williams, K.D., and Karau, S. J. "Social Loafing and Social Comparison: The Effects of Expectations of Co-Worker Performance," Journal of Personality and Social Psychology (61), 1991, pp 570-581. 